Classification

Physics Abstracts

$07.80-61.16 \mathrm{D}-61.80 \mathrm{M}-79.20 \mathrm{~K}$

\title{
Detection and quantification of low energy, low level electron energy loss edges
}

\author{
Alan Richard Wilson \\ Aircraft Materials Division, Aeronautical Research Laboratory, DSTO, 506 Lorimer Street, Fisher- \\ mens Bend 3207, Victoria, Australia
}

(Received September 17, 1990; accepted January 28, 1991)

\begin{abstract}
The detection and quantification of low energy, low level edges presents several problems for Electron Energy Loss Spectrometry. These edges can have very low "jump" ratios making recognition of the presence of the edge difficult. For quantitative analysis the onset energy of the edge must also be identified. Both of these problems can be overcome by the routine use of a second difference (top-hat) filter [1]. The next major problem is that of background subtraction. The standard $a E^{-r}$ curve fit results in over subtraction of the background for spectra of a Li K edge [1]. The same curve constrained to fit the background at high energy as well as low energy can result in under subtraction of the background. Modifying the standard fit to $a(E+m)^{-r-c E}$ and constraining the curve to fit at higher energy shows promise and is easy to implement. The " $c$ " term allows for variation of the power law with energy however the most critical term appears to be the " $m$ " term. For very low level edges another problem is encountered in the subtraction of the background associated with the electron detection system. The detector background level for a yttrium- aluminium- garnet/ Photodiode Array detector has been observed to vary in a non-linear way with incident electron intensity. This affects both the pre-edge background curve fitting and the results of edge count integration.
\end{abstract}

\section{Edge detection.}

Figure 1 shows a spectrum obtained from an aluminium-lithium alloy with nominal composition of 2.2 weight \% (10.5 atomic \%) of Lithium.

The Li edge in this spectrum is always below the pre-edge background intensity i.e. it has a jump ratio less than one. The onset energy of the Li edge and even its existence is difficult to determine from such a spectrum. Processing this spectrum by convolution with a top-hat filter to give the second difference spectrum results in a more reproducible determination of the onset energy as shown in figure 2 . The top-hat filter used should be slightly wider than the resolution of the spectrum so that the minimum preceding the zero crossing corresponds to the energy at which the resolution limited edge commences. A top-hat filter with a wider pass energy lowers the energy of the minimum [1] however this does not affect edge integration results since the counts in the extra energy range will be zero after spectrum background subtraction. 


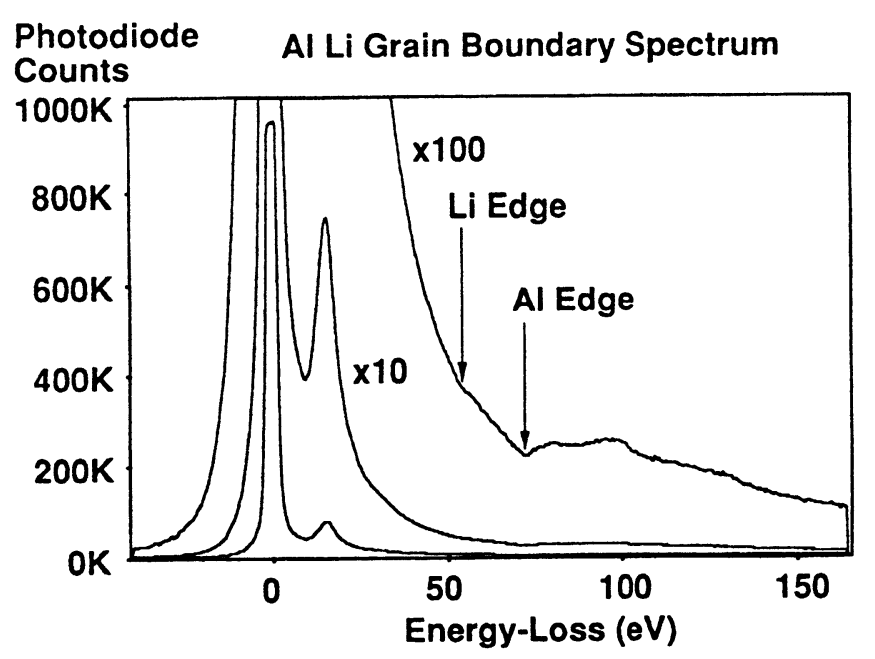

Fig. 1. - Al-Li alloy PEEL spectrum after detector dark-current subtraction. The Li edge has a jump ratio less than one and the edge onset energy is inexact.

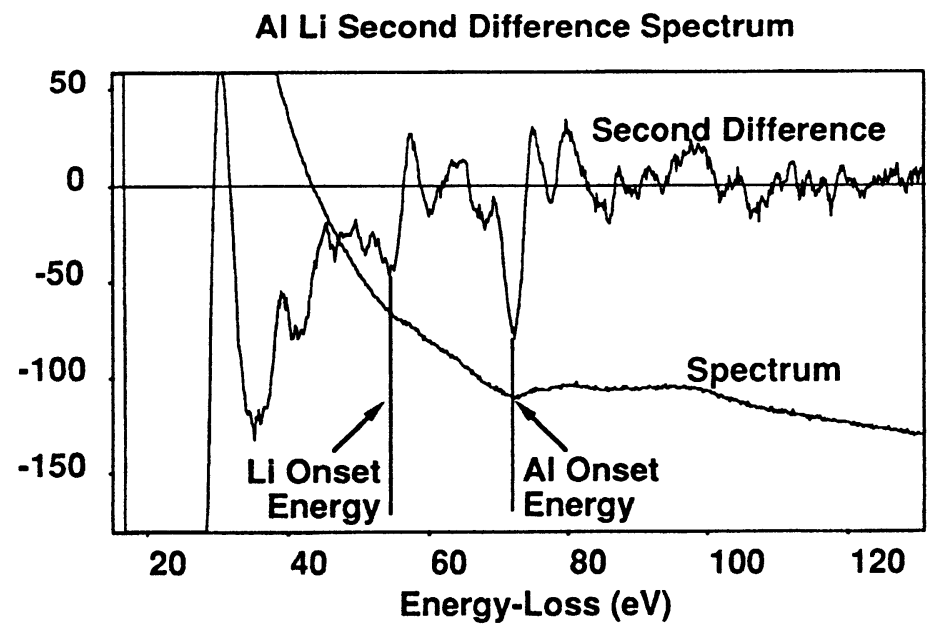

Fig. 2. - The same spectrum as in figure 1 after convolution with a top-hat filter to obtain the second difference spectrum. The presence of the Li edge is more easily detected and the onset energy is determined by the minimum preceding the zero crossing for the $\mathrm{Li}$ edge.

\section{Background fitting.}

In order to quantify the spectrum shown in figure 1 it is necessary to remove the spectral background under the $\mathrm{Li}$ edge. The standard $a E^{-r}$ curve fit to the pre-edge background gave the $\mathrm{Li}$ concentration results shown in table I for a series of spectra obtained successively from points across a grain boundary in this $\mathrm{Al}-\mathrm{Li}$ alloy. 
Table I. - Li concentration with the electron beam placed progressively across a grain boundary in an Al-Li alloy of nominal concentration of 10.5 atomic\% of $\mathrm{Li}$.

\begin{tabular}{|l|l|l|l|l|l|l|l|l|}
\hline Beam Position & 1 & 2 & 3 & 4 & 5 & 6 & 7 & 8 \\
\hline Li at.\% & 3.1 & 2.7 & 2.4 & 3.5 & 9.3 & 8.2 & 5.5 & 0.4 \\
\hline
\end{tabular}

Egerton's [2] results based on the hydrogenic model were used to determine the scattering cross sections and hence the elemental concentrations. Although it was suspected that the $\mathrm{Li}$ segregates to the grain boundary [1-3] (as the relative concentration results in this table indicate) the absolute values of the $\mathrm{Li}$ concentration are lower than the average bulk concentration of 10.5 atomic\%. Incorrect modelling of the background, resulting in over subtraction under the Li edge, is a possible explanation of the low concentration values. Spectra were subsequently collected for a $\mathrm{Li}_{2} \mathrm{CO}_{3}$ sample to examine the effect of different models on background subtraction.

Figure 3a shows a complete $\mathrm{Li}_{2} \mathrm{CO}_{3}$ spectrum while figures $3 b$, $c$ and $d$ show specific parts of this spectrum along with the corresponding $a E^{-r}$ background curve fits. The Li:C:O ratios obtained after background subtraction are 0.8:1:2.9. The good $\mathrm{C}: \mathrm{O}$ ratio implies that this background model is adequate for $\mathrm{K}$ edges with energies above $250 \mathrm{eV}$. The $\mathrm{Li}$ ratio, however, is very low and examination of figure $3 b$ shows that the standard $a E^{-r}$ model badly over subtracts the background under the Li edge. Constraining the $a E^{-r}$ curve to fit the spectrum at higher energies (around 100eV, Fig. 4) for the Li edge results in a very poor background fit. The background curve in figure $3 \mathrm{~b}$ has too great a curvature. A curve of the form $a(E+m)^{-r}+b$ will give a lower curvature for " $m$ " greater than zero and the " $b$ " term will allow for a constant level shift. A slightly different curve of the form $a(E+m)^{-r-c E}$ will allow for the increase in exponent that is expected with increasing energy [4]. Background curve fits using $a(E+m)^{-r-c E}$ or $a(E+m)^{-r}+b$ constrained to fit at higher energies (also shown in Fig. 4) give Li:C:O ratios of 1.6:1:2.9 and 1.5:1:2.9 respectively. These $\mathrm{Li}$ ratios are double those obtained using the simple background model and are closer to the expected value although still too low by $25 \%$. If the same is true for the $\mathrm{Al}-\mathrm{Li}$ alloy then the results in Table I might need to be at least doubled which gives Li concentrations more consistent with segregation to the grain boundary.

Unfortunately these more complicated background models must be constrained to match the spectrum at higher energies. Thus they can not immediately be applied to the Al-Li alloy spectra because of the large $\mathrm{Al} \mathrm{L}_{2,3}$ edge at $73 \mathrm{eV}$. The $\mathrm{Al} \mathrm{L}_{2,3}$ edge can be stripped from the spectrum by extrapolating the background fit to the pre $\mathrm{Al} \mathrm{L}_{2,3}$ edge to higher energy and then the $\mathrm{Li}$ background can be constrained to fit this extrapolated spectrum. This was tried on some of the Al-Li spectra and gave increases in the Li edge integrated counts above those obtained with the standard background fit of 1.63 for a constrained $a E^{-r}$ background and 1.20 for a constrained $a(E+m)^{-r}+b$ background. However, a major source of error is the amplification of the error in the background under the $\mathrm{Al} \mathrm{L}_{2,3}$ edge when it is extrapolated to higher energies. A reduction of $10 \%$ in these extrapolated counts gives a $50 \%$ increase in the Li edge integrated counts for a constrained $a(E+m)^{-r}+b$ curve (i.e. a factor of 1.77 above the standard background result). This indicates that there may be large errors in the determination of the Li concentration due to small errors in the determination and extrapolation of the background under the $\mathrm{Al}_{2,3}$ edge and limits the usefulness of this approach. 


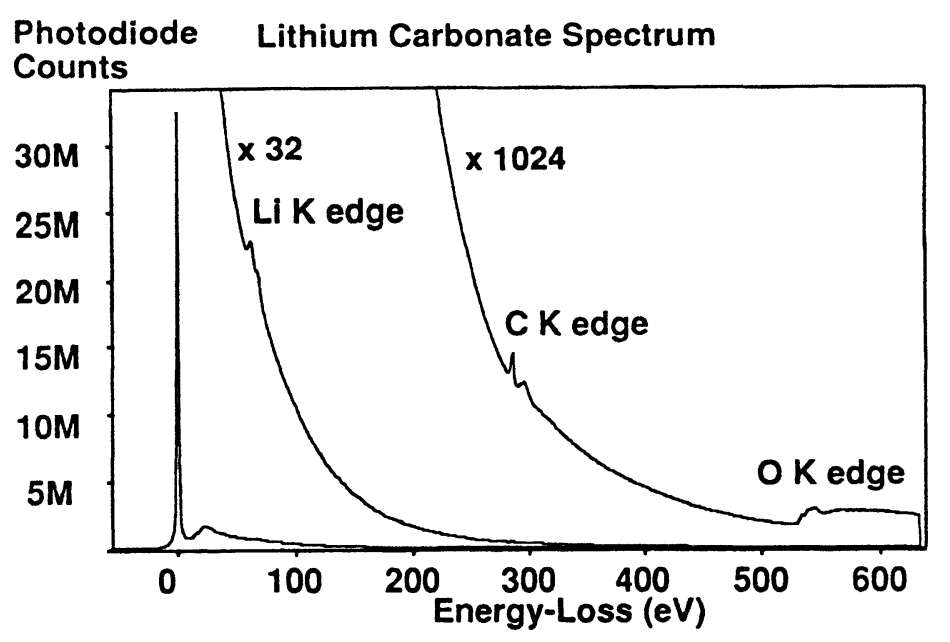

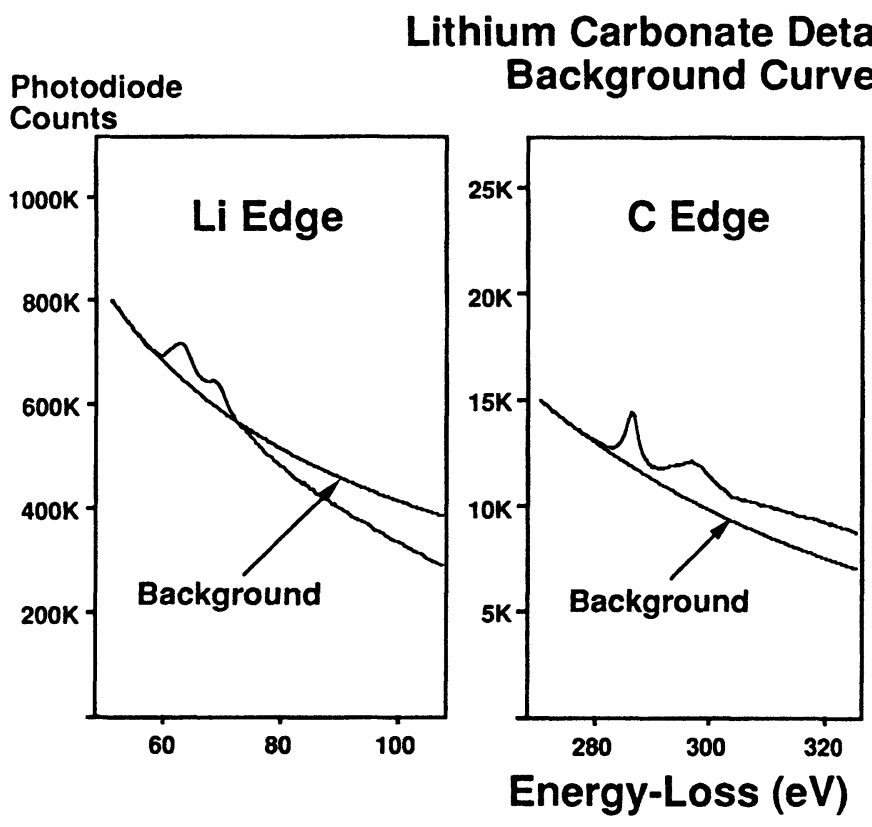

(b)

(c)

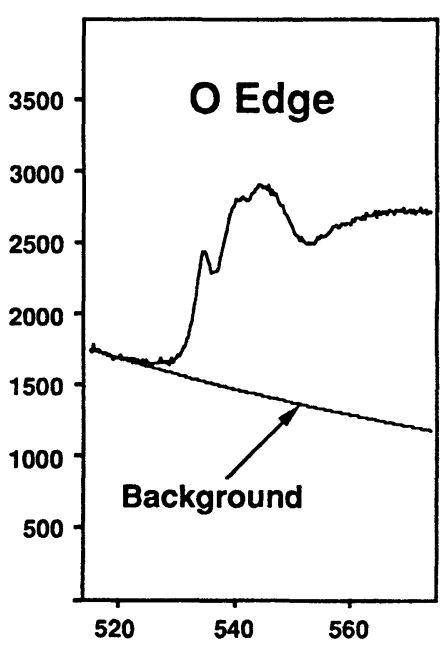

(d)

Fig. 3. - a) $\mathrm{Li}_{2} \mathrm{CO}_{3}$ PEEL spectrum. b), c) and d) show detail around the $\mathrm{Li}, \mathrm{C}$ and $\mathrm{O}$ edges respectively with the corresponding $a E^{-r}$ background curves. The Li:C:O concentration, ratios of 0.8:1.0:2.9 obtained after background subtraction indicates that the standard background curve is adequate for the $\mathrm{C}$ and $\mathrm{O}$ edges but inadequate for the Li edge.

\section{Detector background.}

Fitting curves such as $a(E+m)^{-r-c E}$ to low energy loss background is an empirical approach to background modelling. The " $m$ " term appears to be the most critical parameter. The background fits under the Li edge in the spectrum considered above gave positive values for " $m$ " 


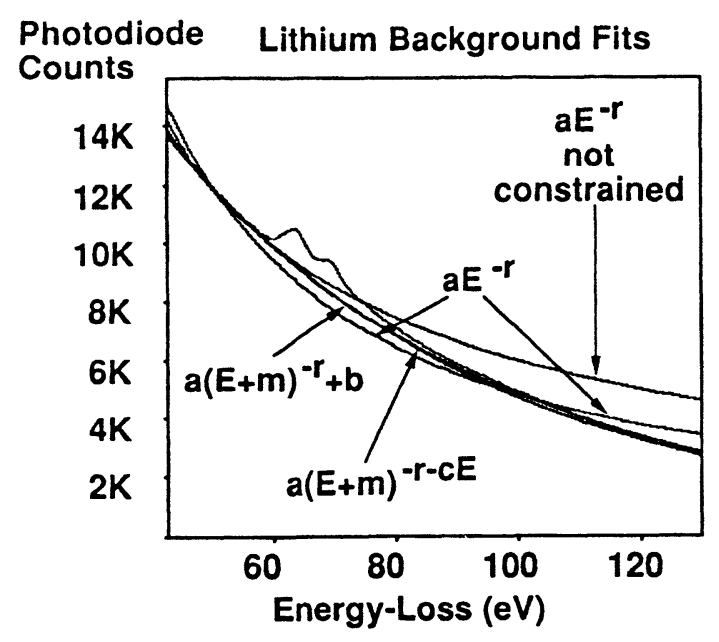

Fig. 4. - Detail around the Li edge in figure 3a for four different background curves with three of them constrained to match the spectrum at higher energies. The two more complicated background curves give superior background fits and similar results for the Li concentration.

which results in a background curve with a lower curvature and also a lower rate of change of curvature than for " $m$ " equal to zero. Since positive " $m$ " improves the post edge background fit this implies that the pre edge background has a higher curvature than is expected for the standard $a E^{-r}$ background model. This higher curvature would occur if the Yttrium- Aluminium- Garnet / Photodiode Array (YAG/PA) detector has a contribution to the background which increases with increasing incident electron intensity. Standard operating procedures of YAG/PA detectors indicates that non-linearity with electron intensity is a problem in two ways: 1) it is common practice to condition the YAG/PA detector with a uniform electron intensity since it exhibits changes in gain dependent on its previous electron exposure history and 2) the YAG/PA detector exhibits electron beam memory, e.g. if an intense zero-loss-peak (ZLP) is recorded then subsequent spectra will have a small peak in the same position of the ZLP even with no incident electron beam.

Data was collected on the Gatan PEELS system with a dispersed electron beam and with a voltage offset applied to the spectrometer to image the edge of the entrance aperture of the spectrometer onto the YAG/PA detector. This gives a range of incident electron intensity in the one "spectrum" as shown in figure 5 and also includes the dark-current signal for the conditions under which the "spectrum" was obtained.

Any shift in the "spectra" due to spectrometer or electron microscope high voltage drift was accounted for by aligning the second difference minima of each "spectrum". In all the cases considered below the dark-current signal was averaged and then subtracted from the counts collected in the four regions of interest shown in figure 5 before any further analysis was carried out. (The dark-current was observed to be uniform, within the noise, over the whole spectrum.) Figure 6 and table II show the effect of increasing the acquisition time on the counts in the four regions of interest. The ratios in figure 6 and table II have been determined by

$$
\operatorname{Ratio}(X \text { Seconds, Region } Y)=\frac{C(X, Y)}{C(1, Y) \cdot X}
$$

where $C(X, Y)$ is the recorded counts for $X$ seconds in Region $Y$. If the counts detected were proportional to the collection time then $C(X, Y)=X \cdot C(1, Y)$ and the ratio in equation (1) 


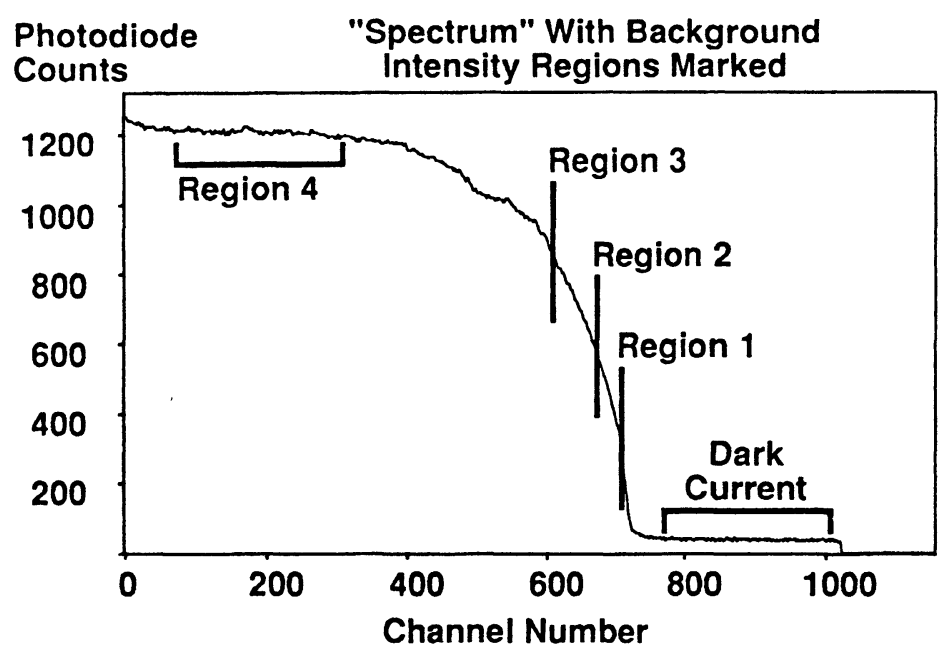

Fig. 5. - Display showing the image of the edge of the PEEL spectrometer entrance aperture. This spectrometer configuration was used to study the YAG/PA detector response in the different intensity regions marked Region 1, Region 2, Region 3, and Region 4.

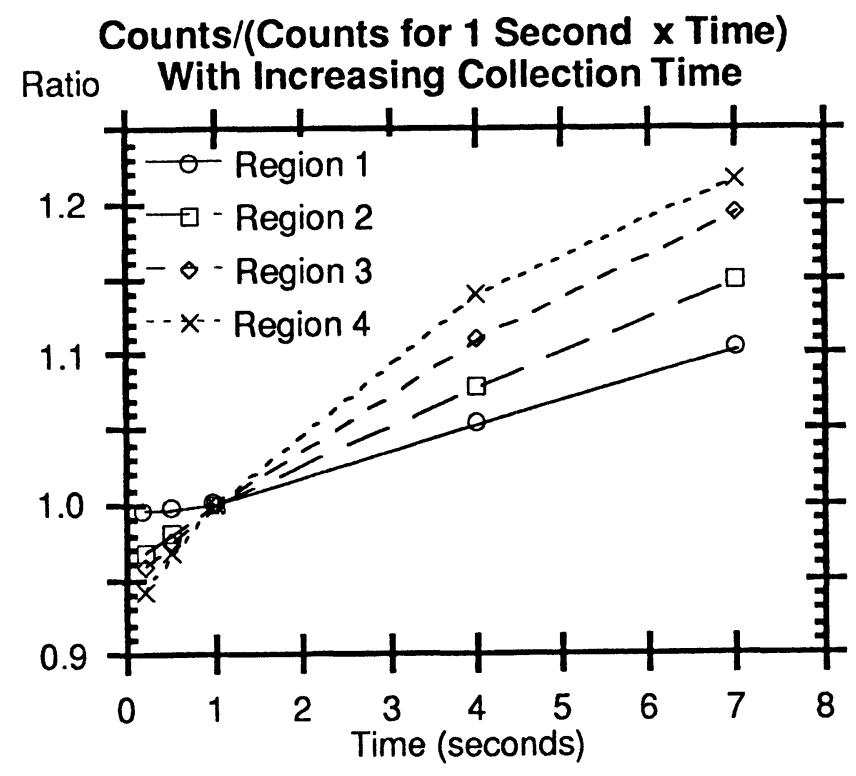

Fig. 6. - Plot of the change in the ratio of counts given by equation (1). The increase in this ratio, for the four different intensity regions defined in figure 5 , indicates that there is a contribution to the detected counts above that expected by the increase in acquisition time. Also, the intensity region with the greatest counts increases most indicating that this extra contribution to the counts depends on the electron intensity striking the YAG/PA detector.

would be equal to one. This ratio is unity if the detector background is constant (i.e. the darkcurrent) or proportional to the total number of incident electrons. In the later case the detector 
Table II. - Dark-current subtracted data for the counts in the regions identified in figure 5 for different collection times. The ratios were calculated using equation (1). If the background is constant (and equal to the dark-current) or is linearly related to the incident intensity then these ratios would all be equal to 1. The ratio data is plotted in figure 6.

\begin{tabular}{|c|c|c|c|c|c|c|c|c|}
\hline $\begin{array}{c}\text { Time } \\
\text { (seconds) }\end{array}$ & $\begin{array}{c}\text { Region 1 } \\
\text { Counts }\end{array}$ & $\begin{array}{c}\text { Region 1 } \\
\text { Ratios }\end{array}$ & $\begin{array}{c}\text { Region 2 } \\
\text { Counts }\end{array}$ & $\begin{array}{c}\text { Region 2 } \\
\text { Ratios }\end{array}$ & $\begin{array}{c}\text { Region 3 } \\
\text { Counts }\end{array}$ & $\begin{array}{c}\text { Region 3 } \\
\text { Ratios }\end{array}$ & $\begin{array}{c}\text { Region 4 } \\
\text { Counts }\end{array}$ & $\begin{array}{c}\text { Region 4 } \\
\text { Ratios }\end{array}$ \\
\hline 0.2 & 69 & 1.00 & 118 & 0.97 & 196 & 0.96 & 283 & 0.94 \\
\hline 0.5 & 173 & 1.00 & 198 & 0.98 & 498 & 0.97 & 725 & 0.97 \\
\hline 1.0 & 347 & 1.00 & 609 & 1.00 & 1022 & 1.00 & 1500 & 1.00 \\
\hline 4.0 & 1459 & 1.05 & 2623 & 1.08 & 4537 & 1.11 & 6840 & 1.14 \\
\hline 7.0 & 2678 & 1.10 & 4893 & 1.15 & 8545 & 1.19 & 12767 & 1.22 \\
\hline
\end{tabular}

background contribution to the counts would be equivalent to increasing the gain of the detector. Figure 6 and table II show that this is not the case and that the count ratios increase with both increasing acquisition time and incident electron intensity. For instance in Region 4 the counts of 1500 for the one second acquisition time scale to 10500 for seven seconds acquisition time if the counts detected were proportional to the acquisition time. The experimental counts of 12767 for the seven second acquisition time are $22 \%$ higher than the scaled counts of 10500 . In Region 2 , a region with lower incident electron intensity than Region 4 , the experimental seven second acquisition time counts are only $15 \%$ higher than the scaled one second acquisition time counts. A similar trend is observed when comparing other regions and indicates that the extra counts over those expected if the counts were proportional to the acquisition time increases non-linearly with the incident electron intensity.

Data was also collected with varying incident electron intensity. The electron intensity was changed by either increasing the electron gun bias or focussing the second condenser lens. These results are shown in figures $7 \mathrm{a}$ and $\mathrm{b}$ respectively plotted as ratios, in a similar way as in figure 6 , but normalized as folows:

$$
\text { Ratio(Setting } X, \text { Region } Y)=\frac{C(X, Y) \cdot C(1,1)}{C(X, 1) \cdot C(1, Y)}
$$

where $C(X, Y)$ is the recorded counts in Region $Y$ for a particular Setting, $X$, of the electron gun bias or the second condenser lens. This normalization is used since there is no absolute measure of the change in incident electron beam intensity and it is assumed that the relative incident electron intensities between the four regions remains constant. If the counts were proportional to the incident electron intensity then

$$
C(X, Y)=k_{y} \cdot C(X, 1)
$$

where $k_{y}$ is constant and equation (2) becomes

$$
\begin{aligned}
\text { Ratio(Setting } X, \text { Region } Y) & =\frac{k_{y} C(X, 1) \cdot C(1,1)}{C(X, 1) \cdot k_{y} C(1,1)} \\
& =1 .
\end{aligned}
$$

Figures 7a and $b$ clearly show that the recorded counts are not proportional to the incident electron intensity but increase with increasing incident intensity and that the background increases 

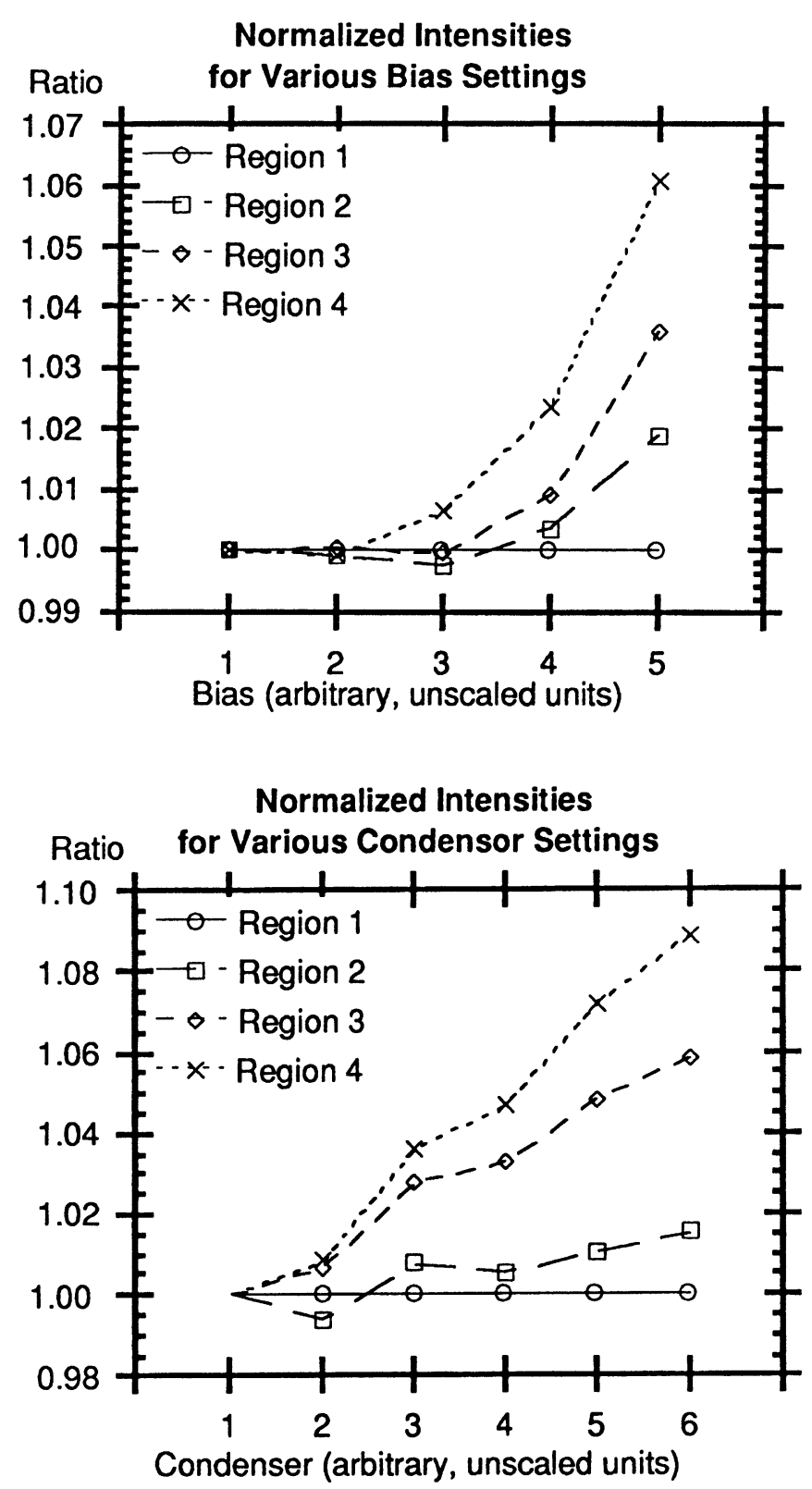

(a)

(b)

Fig. 7. - Plot of the change in the ratio of counts given by equation (2) for a) increasing electron gun bias and $b$ ) increasing second condenser lens focus. The increase in the ratios with the increase in electron intensity in both a) and b) again indicate that there is a contribution to the counts detected above that expected by the simple increase in electron intensity. The higher values for the regions with the higher intensities also indicates that these extra counts are greater for greater incident electron intensity. 
non-linearly with the incident electron intensity. This dependence of the detector background on incident intensity indicates that the effects on a PEEL spectrum will be most severe in regions of rapidly varying intensity. This behaviour has been observed in the $\mathrm{Li}_{2} \mathrm{CO}_{3}$ spectra with the standard $a E^{-r}$ curve proving adequate in the relatively flat regions of the spectra above $250 \mathrm{eV}$ and inadequate for the low energy $\mathrm{Li}$ edge which is sitting on a more rapidly varying background.

The experimental intensity may possibly be related to the true detector background subtracted intensity as follows:

$$
l_{\mathrm{BS}}(E)=l_{\mathrm{Exp}}(E)-l_{\mathrm{DC}}(E)-f\left\{l_{\operatorname{Exp}}(E),\right\}
$$

where $E$ is the channel number of the spectrum, $l_{\mathrm{BS}}$ is the intensity less all detector background counts, $l_{\mathrm{Exp}}$ is the experimentally recorded intensity, $l_{\mathrm{DC}}$ is the dark-current of the detection system and $f\{\}$ is a function which gives the intensity dependent part of the detection system background. Attempts to find a suitable model for $f\{\}$ have not been successful. This failure is thought to be due to the use of count ratios. An initial attempt to overcome this was made by simultaneously collecting X-ray and PEELS spectra from a microtomed aluminium specimen and examining the change of zero-loss and plasmon peak intensity, while varying the electron gun bias, with the assumption that the X-ray counts were directly proportional to the incident electron intensity. Unfortunately the beam has to be spread over a large area of the specimen to obtain sufficient Xray counts while not saturating the PEELS system. This caused a large scatter in the results and it was not possible to determine $f\{\}$. A better approach would be to use a Faraday cup and directly measure the incident electron beam current. This has not been done but is under consideration.

The zero-loss peak intensities, plasmon peak intensities and the ratio of theses two intensities (ZLP/PP), obtained from the aluminium specimen above with varying gun bias, are shown in table III.

Table III. - Zero-loss and first plasmon peak intensities, obtained from a single area of microtomed aluminium alloy, and their ratios, normalized to the lowest bias setting, for increasing electron beam intensity (i.e. increasing electron gun bias setting). The ZLP/PP ratio would be expected to be constant. The increase with increasing electron intensity again implies that the counts recorded by the YAG/PA detector have a detector background component which increases non-linearly with the electron intensity striking the detector.

\begin{tabular}{|c|c|c|c|}
\hline $\begin{array}{c}\text { Increasing } \\
\text { Bias }\end{array}$ & $\begin{array}{c}\text { Zero Loss Peak } \\
\text { Intensity (ZLP) }\end{array}$ & $\begin{array}{c}\text { Plasmon Peak } \\
\text { Intensity (PP) }\end{array}$ & $\begin{array}{c}\text { ZLP/PP Ratio } \\
\text { (Normalized) }\end{array}$ \\
\hline 1 & 54484 & 2651 & 1 \\
\hline 2 & 117650 & 5390 & 1.06 \\
\hline 3 & 219742 & 9791 & 1.09 \\
\hline 4 & 308718 & 13336 & 1.13 \\
\hline 5 & 409591 & 17260 & 1.16 \\
\hline 6 & 506873 & 21126 & 1.17 \\
\hline 7 & 590617 & 24611 & 1.17 \\
\hline 8 & 665218 & 27302 & 1.19 \\
\hline
\end{tabular}


The ZPL/PP ratio increases with increasing electron intensity again indicating that the recorded counts are not simply proportional to the incident electron intensity on the YAG/PA detector. Two other mechanisms which could change the ZLP/PP ratios must be considered. These are mass loss from the specimen and contamination of the specimen. Mass loss would give an increase in the ZLP/PP ratio with increasing exposure of the specimen to the electron beam and thus the spectra collected first in a series of spectra would have lower ZLP/PP ratios than the spectra collected last. The results in table III were obtained from a series of spectra with the electron gun bias initially set to its highest value. The first spectra in this series have higher ZLP/PP ratios than the last spectra collected which is the reverse of what would be expected if mass loss was the cause of the observed ZLP/PP ratio change and indicates that any mass loss occuring is less than the increase in detector background with increase in electron intensity. Contamination of the specimen would result in greater electron scattering, due to the build up of carbon on the specimen surface, and would reduce the ZLP/PP ratio with exposure to the electron beam. In the experiments on the aluminium (results in Tab. III) care was taken to avoid contamination by cooling the specimen in a liquid nitrogen holder and by not using a focussed electron beam. Also, there was no evidence of the carbon plasmon at $22 \mathrm{eV}$ in any of the spectra indicting that there was no carbon build up on the specimen surface. Similar sets of spectra collected with initially low gun bias showed the same variation of the ZLP/PP ratio with electron intensity confirming that contamination was not occuring. Thus the results in table III confirm the results obtained using the image of the spectrometer aperture on the YAG/PA detector and show that there is a contribution to the PEELS spectra from the YAG/PA detector which increase non-linearly with increasing incident electron intensity on the detector.

\section{Possible mechanism for YAG gain variation.}

YAG scintillator material is non-conductive and thus may accumulate an internal charge upon exposure to high energy electrons. This accumulated charge may be considered as a distributed retarding potential for electrons entering the scintillator. For an electron beam with sufficient energy to pass through a thin (uncharged) YAG scintillator disc a retarding potential will result in a greater number of photons produced per incident electron due to the higher interaction of the reduced energy electron with the scintillator material. (For example, the mass range of a $200 \mathrm{keV}$ electron is $40 \%$ higher than that for a $160 \mathrm{eV}$ electron [5]). This will give a greater gain for the YAG/PA detector in the regions of the YAG with an accumulated charge. For electrons that do not have enough energy to pass through the scintillator the total energy available for photon production will be reduced by a retarding potential. This will give a reduction in gain for the detector in the regions of accumulated charge. The thickness of the YAG scintillator to just stop all of the electrons, for different incident energies, can be calculated [5] and is between $35-50 \mu \mathrm{m}$ $(100 \mathrm{keV}), 110-150 \mu \mathrm{m}(200 \mathrm{keV})$ and $200-280 \mu \mathrm{m}(300 \mathrm{keV})$. The experimental results presented in this paper indicate that the YAG thickness in the PEELS used is less than $110-150 \mu \mathrm{m}$.

\section{Conclusions.}

The routine use of a top-hat filter greatly aids in the detection of low level Li edges and is essential for the determination of the edge onset energy. The standard $a E^{-r}$ background curve fit appears to be adequate for $\mathrm{K}$ edges above $250 \mathrm{eV}$ but overestimates the background at lower energies. More complicated curves such as $a(E+m)^{-r-c E}$ or $a(E+m)^{-r}+b$ give better results for low energy (lithium) edges however are not universaly applicable because of the need to constrain 
the curves to fit the spectrum at higher energies. A detailed investigation into the behaviour of the YAG/PA detector with change in incident electron intensity shows that the detector background level increases non-linearly with increasing incident electron intensity. This explains the poor results for the standard background fits at low energy losses since the non-linear detector background will be of most importance in the regions of rapid change in intensity. Calibration of this detector response has proved to be difficult and more work needs to be done in this regard.

\section{References}

[1] WILSON A.R., "Lithium detection in aluminium-lithium alloys using PEELS", Microbeam Analysis, J.R. Michael and P. Ingram Eds. (San Fransisco Press, 1990) p.39.

[2] EgERTON R.F., Electron Energy Loss Spectroscopy in the Electron Microscope (New York: Plenum Press, 1986) $184 \& 357$.

[3] Miller W.S., Thomas M.P., Lloyd D.J. and Creber D., "Aluminium- Lithium Alloys III", Proc. Third Int Al-Li Conf, 1986, The Institute of Metals, London, 584.

[4] EgERTON R.F., Electron Energy Loss Spectroscopy in the Electron Microscope (New York: Plenum Press, 1986) 255.

[5] KANAYA K. and OKAYAMA S., "Penetration and energy-loss theory of electrons in solid targets", J. Phys. D: Appl. Phys. 5 (1972) 43. 